\title{
Hématotropique : Ethnographie, violence et expérience
}

\author{
Simon Latendresse Université de Montréal
}

Résumé : Depuis les trois dernières décennies, les thèmes de la violence et de l'urgence politique ont commencé à se cristalliser comme champ d'intérêt à part entière en anthropologie. Une tendance qui coïncide avec un tournant réflexif qui fait parfois une plus grande place à l'expérience et aux « textures » du vécu par rapport à la systématisation des savoirs. À partir d'une réflexion sur mes propres expériences de terrain au Pakistan et de lectures critiques autour de terrains de la violence, j'explore comment cette approche anthropologique renoue, en lien avec la question de la vérité, les fils de l'épistémologie et de l'éthique-politique. Comment, par son caractère plus engagé, elle pointe vers la possibilité d'un potentiel transformateur de l'anthropologie.

Mots clés : anthropologie; ethnographie; Pakistan; violence; expérience; parrhesia; éthique; politique; biopolitique

Abstract: Over the past three decades, the themes of violence and political urgency have begun to crystallize into an anthropological field of interest in its own right. This trend coincides with the reflexive turn, which sometimes makes more room for the "textures" of lived experience than for the systematization of knowledge. Starting from a reflection on my own experiences in the field in Pakistan and drawing on critical readings about fieldwork experiences of violence, I explore how this anthropological approach weaves anew the threads of epistemology and ethics-politics around the question of truth. I show how, through its more engaged character, this approach points towards the possibility of a transformative anthropology.

Keywords: anthropology, ethnography, Pakistan, violence, experience, parresia, ethics, politics, biopolitics

420 / Simon Latendresse
Au temps d'Homère, l'humanité s'offrait en spectacle aux dieux de l'Olympe: c'est à elle-même aujourd'hui qu'elle s'offre en spectacle. Elle s'est suffisamment aliénée à elle-même pour être capable de vivre sa propre destruction comme une jouissance esthétique de premier ordre.

Benjamin (2000 [1939], 313).

$\Lambda$ rrivé au Pakistan pour la première fois en 2006, c'est à Ala suite d'un parcours par le monde du développement et de l'aide humanitaire dans ce pays que j'ai effectué, à partir de 2009, un virage vers l'anthropologie. J'entame ce texte par ce détail biographique puisqu'il implique ici le contexte dans lequel les détails ethnographiques qui suivent ont été puisés: lors d'un travail de terrain de nature militante plutôt qu'académique, même s'il s'agissait d'une démarche d'insertion et d'observation de longue haleine. Ces observations, de même que cet apport mutuel entre les démarches activistes et anthropologiques, me paraissent néanmoins aujourd'hui d'une pertinence de premier ordre pour formuler une réflexion autour de la question d'une anthropologie de la violence, située au carrefour de la science et de l'engagement politique. Une anthropologie du trouble, autant qu'une anthropologie troublée par une constante tension éthique et épistémologique, et du même souffle, entre savoir et action.

\section{Deux corps}

On l'a repêché des eaux de l'Indus il y a à peine quelques heures. C'est le seul qu'on a retrouvé de deux garçons disparus, il y a presque une semaine. Le corps gît au milieu d'une hutte communautaire où s'entassent, sous une chaleur étouffante, les hommes du village. La pièce exigüe empeste l'odeur de putréfaction avancée et celle du camphre qui, même en quantités excessives, parvient mal à masquer la première.

Comme de nombreux jeunes hommes du village, il plongeait tous les jours au pied du barrage de Taunsa pour repêcher et revendre la ferraille laissée par les travaux de remodélisation ${ }^{1}$. Cette mort évitable témoigne 
des risques de plus en plus grands que doivent prendre ces jeunes pêcheurs pour gagner leur vie. Mourir pour survivre? Des sentiments de la foule, il est difficile de savoir lequel du deuil ou de l'indignation l'emporte. J'y suis avec ma caméra. Je suis sur place depuis plusieurs mois pour tourner un documentaire sur cette communauté de pêcheurs adivasis ${ }^{2}$ - plus particulièrement sur leur lutte sociale contre le mégaprojet en cours sur le barrage - et aujourd'hui ils m'ont expressément demandé de filmer les funérailles. Et pourtant, un sentiment de pudeur et de respect pour la famille m'empêche d'empoigner l'appareil pour capter de telles images. Je reste en retrait, sentant que je n'ai rien à faire là. Je sens ma seule présence comme presqu'indécente. Malgré tout, un membre de la communauté me prend par le bras, à ce moment précis, tasse quelques-uns des proches rassemblés devant la dépouille pour m'amener droit devant et me prie de filmer la scène. J'ai encore sur cassette les images de ce cadavre violet et boursoufflé que je n'ai jamais pu me résoudre à inclure au montage ${ }^{3}$.

Autre moment (même lieu, même tournage). Malgré les tentatives d'intimidation explicites à notre endroit de la part d'un cacique local, quatre hommes du village insistent pour m'emmener tourner quelques prises et recueillir les témoignages du meurtre d'un des leaders du mouvement local Sindhu Bachao Tarla (« Mouvement 'Sauvez l'Indus' ») $)^{4}$. Ici pas de cadavre. Seules les taches de sang, encore visibles sur le pont du bateau où l'incident est survenu, rend quelque peu plus palpable cette menace qui jusqu'alors n'était demeurée que verbale. Un témoin oculaire parle à la caméra, sans chercher à se cacher. Il raconte en détail la scène avec un mélange déstabilisant d'intensité tragique et de détachement émotionnel.

Ces deux scènes se déroulent au sud-ouest du Pendjab pakistanais, au cours de l'été 2007. La guerre civile larvée qui secouera le pays durant toute une décennie ne fait tout juste que commencer (il y a quand même déjà eu deux attentats-suicide dans la capitale depuis mon arrivée). Létat d'urgence n'a pas encore été mis en place par un Général-Président Musharraf en fin de règne et nous n'avons encore qu'une idée plus ou moins abstraite du tumulte qui attend le pays. Nous ne sommes pas en zone de guerre. Pas encore. Pourtant, ces communautés de pêcheurs de l'Indus subissent chaque jour les expulsions massives, la paupérisation extrême, l'intimidation policière et les assassinats, en plus de l'extorsion de la part de « contracteurs en pêcheries » qui se sont appropriés le fleuve. La violence quotidienne de la paix fait se demander quelle différence il pouvait bien y avoir avec la guerre.

...Elle me fait aussi souvent me demander: «Qu'est-ce que je peux bien faire ici? ».

\section{Entre banalisation et fascination ?}

De quel droit? À quoi, mais à qui, surtout, pouvait bien servir ce documentaire militant en voie de se transformer en film ethnographique? Mon beau projet, de toute évidence, n'apporterait que bien peu de réconfort à ces gens méprisés par les élites terriennes, et que la Marche de l'Histoire pakistanaise s'efforçait d'écraser. Ces gens vus par une bureaucratie urbanisée comme des « reliques» d'un passé nomade, des obstacles à la « modernisation ".

Mais qu'est-ce que je pouvais bien faire là?

De 2007 à 2009, la situation politique, suivant la déclaration de guerre du $\mathrm{TTP}^{5}$ contre le gouvernement, empirait de jour en jour. Pourtant, il n'était pas question pour moi de rentrer au Québec. Je me surprenais même à chercher à m'enfoncer dans des zones plus risquées encore, où régnait un véritable état de guerre civile. D'une part, tout se passait comme si la violence inouïe dans laquelle nous vivions tous devenait, de façon perverse, la condition normale de notre existence. J'écris notre, puisqu'elle faisait indubitablement partie aussi de la mienne. Mais de façon plus troublante encore, peut-être, cette violence exerçait sur moi un pouvoir d'attraction presqu'irrésistible - sans pour autant parler de jouissance. Je voulais y être, je voulais tout voir.

Il s'agit d'une ambivalence dont les reporters de guerre, déjà avant les ethnologues et certains travailleurs humanitaires, ont souvent fait état, et que plusieurs décrivent comme une forme d'addiction. Une dépendance au risque, souvent critiquée avec une certaine légitimité d'ailleurs, comme une recherche de sensations fortes ou une fascination morbide pour le tragique - un tragique surtout et évidemment tiers-mondiste. Cette ambivalence nous pousse à nous questionner à chaque moment sur la validité de notre entreprise: quelle légitimité pour une ethnographie de la violence? Mais aussi, en-deçà des considérations éthiques, en-deçà et au-delà du malaise, de ce conflit interne de l'ethnologue, une interrogation encore plus fondamentale peut-être: quel savoir, quelle "vérité » se révèle du fil qui noue ici la pratique ethnographique, en tant qu'expérience, à l'acte politique, sur ce terrain de l'urgence et de la terreur?

\section{Anthropophagie et parrhésie}

\section{Le voyeur}

Fouillant dans les archives historiques sur les atrocités commises par les colons durant le boom du caoutchouc en Amazonie, au début du XXe siècle, Michael Taussig (1987) affirme avoir été, lui aussi, troublé par cette irrépressible attraction morbide. Dans une entrevue au New York Times, Taussig admet: 
Les faits étaient si horribles, si brutaux et pourtant, je voulais en voir plus. Je devenais comme une sorte de junkie de la violence. Je voulais voir le matériel [d'archive] devenir plus fou et plus violent, et j'ai commencé à me demander "mais qu'est-ce qui se passe avec moi? Est-ce que je suis une si mauvaise personne? ». (cité in Eakin 2001).

On pourrait sans doute marquer au alentours des années 1980-1990 le moment où une anthropologie de la violence s'est réellement cristallisée comme un champ d'enquête spécifique (Schmitt et Schroeder 2001). Une tendance qui a certainement à voir avec l'explosion de conflits armés complexes qui, depuis la chute du rideau de fer, a enflammé les états post-soviétiques et surtout les mondes post-coloniaux, terrains traditionnels de l'anthropologie ${ }^{6}$. Qu'on pense aux travaux de Liisa Malkki (1995) et de Mahmoud Mamdani (2001) sur le génocide rwandais, à Achille Mbembe $(2003,2018)$ qui, en réponse post-coloniale au concept de biopolitique, développe celui de «nécropolitique ". Sans oublier Michel Agier (2008, 2012), en France, qui lève le voile sur les différentes formes de violence imposées aux réfugiés du monde, mais également sur l'expérience d'engagement politique de l'anthropologue sur des terrains de conflit (Agier 1997).

Certains anthropologues comme Mark Duffield (2000) se sont tournés vers l'économie politique ou les approches de types système-monde pour aborder la question des conflits politiques contemporains. Michael Taussig est, toutefois, certainement un des plus célèbres à avoir véritablement « anthropologisé » la question de la violence. Sans jamais la réduire à quelque essentialisme ou atavisme, il fouille plutôt les fondements historiques et symboliques de la terreur (Taussig 1987), en interroge les effets actuels sur la culture, sur le sens que se font les sociétés d'elles-mêmes (Taussig 1992), etc. Sans oublier les répercussions de cette violence sur l'anthropologue lui-même: répercussions psychologiques, d'abord, puis l'impact de cette violence sur sa manière de pratiquer, ses méthodes, sur sa conception de son rôle d'anthropologue (Taussig 2003). Plus près de chez nous, des ethnologues ont aussi commencé à s'interroger au carrefour de l'histoire, de la culture et de la politique pour explorer de plus près l'épidémie de violence et de suicide qui frappe les Autochtones (Bujold 2006 ; Viventi 2011), investissant un champs jadis laissé aux criminologues et psychosociologues?.

Dans cette discipline, à laquelle on avait reproché de projeter hors de l'histoire des pans entier de l'humanité, ce passage de l'intemporel à l'urgence, semble peut-être le bienvenu. Mais cette anthropologie doit aussi répondre, une fois de plus, aux feux d'une critique acerbe qui tend à assimiler cette pratique à une sorte de voyeurisme. Tourisme du tragique qu'exacerbe un rapport de pouvoir global inégalitaire. Excroissance savante « d'un Occident sauvagement anthropophage ", comme l'écrit Natacha Giafferi:

La démarche ethnographique, lorsqu'elle tente de s'appliquer à des populations que l'on a privées des moyens d'une participation réelle à leur histoire comme à celle de l'humanité, est devenue d'une pratique difficile, parce qu'elle y est contestée mais aussi parce que, telle qu'elle a été et est parfois encore menée, elle reste parfaitement contestable. S'il y a une inégalité de fond dont les chercheurs en sciences humaines ne sont pas responsables, il existe aussi dans cette discipline une tradition, bien connue mais plus guère évoquée depuis les confessions de Leiris, de pillage intellectuel joint à une morgue que ne contredit pas un certain compassionnisme coupable. (Giafferi 2004, 123)

Une violence structurelle se doublerait ainsi d'une violence de la relation ethnographique en tant que telle. Il n'est pas rare que de telles critiques soient même souvent formulées par les anthropologues eux-mêmes, voire envers leur propre travail. Au moment de procéder à la description d'un cas particulièrement difficile, Mariella Pandolfi, dans un article intitulé "Théâtre de guerres: passions politiques et violences ", marque une pause toute réflexive, révélatrice de toute l'ambivalence qu'oblige au minimum une attitude autocritique dans l'ethnographie de la terreur: "Pendant que j'essaie de trouver les mots pour décrire cette histoire, je continue à me demander: en ai-je le droit? » (Pandolfi 2008, 103).

"Photographier la catastrophe ", d'Abigail Solomon-Godeau lance une critique similaire envers la tendance des photoreporters à « s'approprier les tragédies humaines, à les exploiter à leur profit en misant sur le voyeurisme ambiant, bref, à faire d'épisodes dramatiques vécus par des hommes des objets marchands. » (Solomon-Godeau 2010, 60). Au sujet de la torture médiatisée d'Abou Ghraib, Isabelle Lasvergnas écrit:

\section{[...] obscénité de la violence et obscénité du sexuel sont devenues confondues [...] indiscernables l'une de l'autre chez le sujet occidental qui n'a cessé de faire reculer en soi les frontières de la honte, et qui du même coup fait subir à l'autre ce même franchisse- ment dans une déréalisation de la violence auquel il le soumet. (Lasvergnas 2007, 120)}

Étranges et inconfortables résonnances avec une certaine pratique contemporaine de l'anthropologie, et particulièrement avec l'ethnographie de la violence et de la terreur. C'est en réfléchissant sur ce genre de critique du sensationnalisme que je me suis moi-même souvent interrogé sur le bien-fondé de ma démarche dans les 
zones de conflit du Pakistan. D'abord, à l'époque où j'y travaillais au sein d'une ONG, frustré par le peu de résultats tangibles que paraissait donner mon travail. Et plus encore, peut-être, alors que je laissais finalement tomber la prétention d'être "utile » et que je me convertissais à l'anthropologie, alors même que le pays étant en proie au terrorisme. Mais pourquoi? En ai-je le droit?

Bien entendu, le travail de reporter, ou même de documentariste, et celui d'anthropologue sont des métiers bien distincts. Les premiers se pratiquent avant tout dans l'instant. Ils existent pour et par l'attention immédiate dans un marché spectaculaire qui ne tolère aucun silence. S'ils sont sans contredit essentiels pour informer et mettre en lumière des évènements qui sont d'intérêt public, c'est une vulnérabilité constitutive que la leur d'être susceptible au sensationnalisme et au voyeurisme.

Lanthropologie existe, pour sa part, dans une temporalité tout à fait différente. Non tributaire de l'instantané médiatique, l'anthropologie (oui, je l'écris ici de manière excessivement schématique) ne se voudrait-elle pas, au contraire, à la recherche de constance, de ce qui, chez l'être humain, transcende, justement, le temps? Les questions de transformations sociales, par exemple, particulièrement chez les sociétés dites traditionnelles, intéressent depuis toujours les anthropologues, mais ne s'agit-il pas alors d'une toute autre échelle de temps que celle de l'urgence humanitaire ou de la crise politique? Mais peutêtre existe-t-il aussi en anthropologie l'ambition inavouée d'en émanciper la littérature, de réputation aride, de son lectorat académique et marginal, et d'attirer un public plus large en misant sur des sujets chauds, attisant l'attrait populaire pour la violence, la guerre, le tragique? Lanthropologie n'évolue-t-elle pas, elle aussi, après tout, dans le même monde désertifié de l'idéologie marchande qui ne conçoit l'utile que dans un rapport d'immédiateté et de profits rapides? En somme, elle survit, comme tout le monde, au rythme des ventes et du capital de l'attention.

Ou encore l'anthropologie d'aujourd'hui baigne-t-elle tout simplement, suivant l'air du temps, dans un sentiment d'urgence généralisée? Cette préoccupation provient certainement le plus souvent d'indignation et d'empathie authentique. Mais en exposant cette violence - parfois de façon très graphique - l'anthropologue se fait-il/elle, lui/ elle aussi, en quelque sorte, complice de cette déréalisation? Ne force-t-on pas l'autre à franchir une « frontière de la honte »? Voilà qui nous amène inévitablement à nous questionner nous amène inévitablement à nous questionner quant à l'attrait que ce genre de terrain exerce sur l'ethnologue lui/elle-même. Sur cet étrange appel que suscite la violence. Comme s'il s'agissait d'une recherche obscène du rush d'adrénaline... Double mouvement d'exorcisme et de renforcement d'un ennui occidental? Une sorte de parcours thérapeutique sous couvert de science et de solidarité tiers-mondiste en forme de sport extrême?

\section{Le corps exposé, le corps du martyr}

Pourtant, sur le terrain, je ne me suis jamais vu moimême rejeté de façon univoque, pas plus que mes interlocuteurs ne semblaient ressentir mon enquête comme une intrusion, encore moins une violation. Tout au plus, certains esprits plus cyniques se moquaient de ma démarche, qu'ils trouvaient foncièrement inutile. Mais comme j'ai eu souvent l'occasion de le constater, cela même que l'anthropologue se gênerait à montrer — voire à regarder lui/ elle-même en face - n'est pas nécessairement objet de censure pour son sujet. Au contraire son exposition peut devenir justement l'objet d'une revendication politique ${ }^{8}$. Ainsi, loin d'être entendu comme une désacralisation ou un défaut de pudeur, montrer le cadavre du noyé dans toute son abjecte nudité, sa mort témoignant d'une injustice, cet acte devient ici un moment de parrhésie.

La parrêsia, nous enseigne Foucault (2004), c'est la «parole vraie », la parole courageuse. Une parole pour laquelle, à l'inverse de la séduction rhétorique, l'orateur encoure un risque. Parfois au péril de sa propre vie. Une parole ou, par extension, me permettra-t-on ici d'inclure l'acte de montrer, ou de se montrer. Parrhésie du corps exposé.

Ici c'est donc l'opprimé, " celui qui n'a que ses larmes » (Foucault 2004, 124), qui par le corps du martyr, le corps mutilé cherche à dire, à montrer l'injustice à travers la lentille de la caméra ou du récit ethnographique. Plus important encore done, c'est ici qu'il se fait sujet non plus seulement au sens de sujet anthropologique mais bien comme agent de l'acte parrèsiastique. La distinction entre reporter et anthropologue se brouille et perd peut-être un peu en importance: vu sous cet angle, son rôle dans cette relation est avant tout celui d'un double intermédiaire. Ce dernier, en effet, par sa situation d'étranger, d'intervenant externe, universitaire, se voit naturellement identifié à l'Occident ou à l'élite urbaine. Pourtant, lorsqu'il a pu gagner, et de longue haleine, la confiance de ses interlocuteurs, ceux-ci verront en sa présence l'espoir, si infime soit-il, de se faire entendre par ces "puissants " auxquels eux n'auront jamais accès. La présence de l'ethnologue - dont le rôle est souvent confondu avec celui du reporter ou de l'activiste - paraît présenter pour eux une opportunité. Une sorte de porte-voix. N'est-il pas alors un peu hâtif de supposer a priori une telle pudeur chez le sujet interrogé, que l'exposition de son corps violenté ne puisse être autre chose que trahison et marchandisation? Ce corps exposé peut, au contraire, faire office de signifiant fort, signe d'un acte parrèsiastique duquel l'ethnographe se fait tout à la fois médium et co-énonciateur. 
La scène qui ouvre ces pages présente assez littéralement l'exposition d'un corps en veille, d'un cadavre. Mais on peut tout aussi bien penser aux blessures, aux corps mutilés, voire à une grève de la faim. On ne fait ici jamais que laisser voir. On met en scène. On fait parler ce corps. On le fait hurler. Qu'on pense aux estropiés iraniens de la guerre Iran-Iraq, aux blessures et aux cicatrices que montrent les survivants des pogroms du Gujarat de 2002, aux vidéos qui pullulèrent sur Youtube montrant le cadavre de Neda Agha Soltan, froidement abattue en pleine rue à Téhéran lors des manifestations de 2009; aux manifestants égyptiens tombés sous les balles, devant les caméras des téléphones portables, lors $\mathrm{du}$ « Printemps arabe ». Au corps du tristement célèbre petit Aylan Kurdi, échoué sur la grève méditerranéenne en 2015; ceux d'un père salvadorien et son enfants, morts sur les rives du Rio Bravo. Images brandies comme témoignages de l'extrême violence que subissent les migrants... Il s'agit bien d'en faire une sorte de spectacle, une image-choc qui fait réaliser comme obscène, non pas l'exposition de l'image elle-même, sinon la violence qui la met au monde.

Loin de n'être que du domaine médiatique, ces images et témoignages, verbaux ou corporels, fournissent justement une matière de premier plan pour une anthropologie qui veut faire sens de l'(in)humanité contemporaine. Pourquoi donc l'anthropologie, sous couvert de respect à une dignité du sujet autrement constamment piétinée, devrait-elle se cantonner dans une sorte de consensualité apolitique? La temporalité de l'anthropologie nous permet justement, au contraire, de saisir ce moment de l'urgence et de travailler plus en profondeur l'évènement, d'entrer dans le fin grain du vécu. Comme l'écrit Chowra Makaremi, sur le terrain de la violence, l'anthropologie

\section{[...] permet deux opérations particulièrement heu- ristiques: d'un côté, entrer dans le détail de la vie quotidienne et de la routine dans des situations où l'exception devient la règle; d'un autre côté, offrir à la dimension subjective la part qui lui revient, en se don- nant les moyens d'examiner les émotions politiques et les visions du monde à travers lesquels la violence est vécue et produite. À travers un jeu d'échelles qui articule les dimensions politiques et intimes, l'ethnographie explore la place accordée dans l'action aux croyances, aux visions du monde, aux cadres de perception et aux systèmes moraux, et s'intéresse aux formes psychiques d'intériorisation des conflits. (Makaremi 2016, 104.)}

Mais au-delà de la place de l'anthropologue, de la légitimité ou de l'utilité de son engagement sur ces terrains, je me questionne également sur la fonction — narrative, politique, juridique? — qu'accomplissent ces images de violence $^{9}$. En quoi il devient si crucial de les montrer? Qu'y a-t-il donc à y trouver au-delà de l'affect, de la pure valeur de choc?

On pourrait voir dans l'exhibition du corps violenté une expression caractéristique du paradigme biopolitique dans lequel nous vivons. D'une forme d'expression propre à cette gouvernementalité moderne dont les pratiques, les discours et les codes passent, ou s'exercent, par et sur le corps - par la gestion de la vie humaine sous la forme massifiée et biologisée de la population (Foucault 1976 ; 2004). Il est peu étonnant, dans ce monde d'hôpitaux, de cliniques, de politiques migratoires et de santé publiques, de statistiques d'emploi, un monde de protocoles sanitaires, de facteurs de risque et de camps de réfugiés, que le pouvoir souverain soit contesté à travers ce même corps déchu sur lequel il s'exerce (Foucault 1976): ce qu'Agamben appelle une « biopolitique mineure » (Grelet et Potte-Bonneville 2000). Ainsi à un pouvoir qui confond incessamment le public et le privé, l'exposition du corps, la grève de la fin, les barrages humains, etc. sont autant de façons de revendiquer ou de résister, politisant justement ce qu'on a de plus intime ${ }^{10}$.

Mais encore! Cette expression politique du corps, qu'on décrit ici comme biopolitique, ne devrait-elle pas alors être circonscrite, géographiquement et historiquement au développement de ce mode de gouvernement " naturaliste », quasi-scientifique (Foucault 2004), comme effet et artefact de celui-ci? D'une part, les principes du libéralisme et du gouvernement biopolitique, comme la sécurité et le "droit à la vie » ne sont jamais, non plus, complètement étrangères aux Pakistanais ou aux Indiens. Pas plus qu'aux Africains. Lorsqu'Achille Mbembe (2003) décrit ce qu'il appelle " nécropolitique » — l'exercice du pouvoir par l'administration de la mort - non seulement sa thèse est une réponse post-coloniale à la théorie de la biopolitique, mais les pratiques qu'il donne en exemple (périmètres "sécuritaire ", blocus économiques, destruction d'infrastructures civiles, insécurité organisée) s'appuient pour la plupart sur le renversement des même méthodes gestionnaires, des mêmes principes, de la gouvernance biopolitique.

Aussi, longtemps avant la naissance de la biopolitique et son hégémonie globale, que Duffield (2000) appelle la "paix libérale ", on ne manque pas de trouver dans nombre de traditions des formes d'expression politique passant ainsi par ce « corps déchu». Le tragique, comme expérience constituante d'une subjectivité politique, le martyr comme expression d'une revendication morale et politique, n'ont rien de phénomènes strictement modernes et encore moins, d'un monopole de la tradition occidentale. La pratique indienne (millénaire) 
de la grève de la faim, justement, est mentionnée dans Le Gouvernement de soi et des autres, comme exemple d'expression parrèsiastique, par laquelle l'individu sans pouvoir, "sans voix ", use de son propre corps, seule arme symbolique qu'il possède, pour dénoncer l'injustice (Foucault 2008). La tradition de l'Islam chi'ite met également l'accent sur l'exhibition ritualisée du corps lacéré et du sang non seulement comme signifiant spirituel, mais aussi comme marqueur théologico-politique, affirmant ou contestant la légitimité d'un pouvoir, d'un statut, politique et historique ${ }^{11}$. Lorsque les activistes du mouvement "Sindhu Bachao » entreprennent de protester contre la Banque mondiale par une grève de la faim illimitée, on peut sans doute affirmer que cet acte prend simultanément racine dans une tradition théologico-politique ancestrale et dans une conception moderne du droit à la vie et de la responsabilité gouvernementale à son endroit.

En 1928, Walter Benjamin (1985) se posait une question similaire, interloqué par l'importance de « ces scènes d'horreur et de martyr où se délectent les drames baroques allemands ». À la fois énigmatique et étrangement révélatrice, Benjamin offre comme un fragment de réponse à travers cet extrait d'un texte sur Ménestrier (Benjamin 1985, 234): "Integrum humanum corpus symbolicam iconem ingredi non posse, partem tamem corporis ei constituendae non esse ineptam ${ }^{12}$ ». «Un corps humain entier ne peut entrer dans une icône symbolique. Une partie du corps (seulement) est plus appropriée pour constituer tel icône. » (234.). Mais Benjamin en fait une lecture idiosyncratique qui irait plutôt dans ce sens: un corps intègre n'a pas le pouvoir symbolique qu'il faut pour faire icône. " [L]e corps humain », précise-t-il, " ne pouvait faire exception à la loi qui commandait de réduire en pièces l'organique afin de recueillir dans ses débris la signification vraie, fixé et scripturaire. » (234.)

Scripturaire. Le corps, ce débris organique, comme inscription. Et si c'est ultimement sur ce corps, comme entité biologique, que s'exerce le pouvoir souverain, et ce depuis même l'antiquité comme l'affirme Agamben (1993), c'est donc seulement par cette cassure biologique elle-même qu'il peut ultimement être contesté (Agamben 1993 ; Foucault 1976). Ce corps du martyr, corps mutilé, corps démembré, devient l'inscription qui dénonce le manquement du souverain ou remet en question sa légitimité, en appelant d'une justice divine ou naturelle. Ce corps est donc toujours un objet politique, voire même

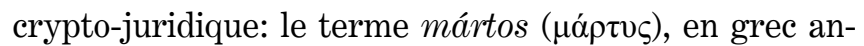
cien - màrtyras ( $\mu \alpha \dot{\rho} \rho \tau \nu \rho \alpha \varsigma$ ) en grec moderne, tout comme l'arabe chahîd (شهيد) - signifient « témoin ». Mais il doit toutefois, pour témoigner de la violence du souverain, pour atteindre ce statut d'inscription, de signifiant politique, en porter sur lui la marque. Qu'on expose au grand jour le cadavre boursoufflé du noyé ou qu'on ne nous montre que les taches de sang incrustées dans le bois du bateau... Ce qui fait sens, ce qui fait «icône » c'est toujours en fait un reste.

Le siècle du Trauerspiel dont parle Benjamin est aussi celui de Descartes. En dépit de son origine religieuse, le drame baroque reflète malgré tout l'esprit rationaliste de son temps. Suite à son exégèse sur Ménestrier, Benjamin met ainsi en lumière le lien arcane qui unit l'esprit cartésien à l'obsession du Trauerspiel pour la violence. Et remarquablement, ce passage résonne aujourd'hui, plus que jamais:

\footnotetext{
Puisque en effet l'esprit est en soi une pure raison, fidèle à elle-même, et puisque seules les influences corporelles le mettent en contact sensible avec le monde extérieur, la force des tourments qu'il subit apparaissait plus facilement comme un fondement de la violence des affects que ce qu'il est convenu d'appeler les conflits tragiques. (Benjamin 1985, 235)
}

Aujourd'hui, dans un esprit similaire, l'empire de l'individualisme méthodologique et de l'homo œeconomicus ramène à la surface ce corps du martyr comme ressort dramatique primordial d'une biopolitique qui, de par la froideur même de sa rationalité gestionnaire, n'en est que plus dépendante encore de la pure physicalité. D'un rapport à l'organique comme seul support tangible sur lequel puissent reposer les rapports politiques. Le slogan féministe «le privé est politique » recouvre désormais ici un sens inédit. Il ne resterait à l'humain pour se faire entendre qu'un cadavre violet et boursoufflé, ou celui troué de trente-six balles de AK-47, laissé près du même village. Le sang qui marque le bois du pont. Il ne suffit pas de savoir qu'il y a violence, qu'il y a injustice. Pour aspirer à signifier politiquement, il faut rendre directement sensuelle la réalité de sa mise-à-mort. Il faut qu'on puisse le voir, le toucher, pour véritablement déranger notre sensibilité biopolitique ${ }^{13}$.

J'écris «voir » et «toucher », comme si ces sens étaient directement communicables... Pourtant, hormis dans la tradition de l'anthropologie visuelle et du film ethnographique, c'est le plus souvent par le biais de la parole, par l'écrit, que les choses se donnent à « voir ». Même pour l'anthropologue lui/elle-même, cette expérience de la violence est généralement absorbée de façon indirecte: à travers les restes, les traces, (un corps, des taches de sang) mais avant tout à travers le témoignage, le travail de mémoire. Un travail qui pose toujours comme ouverte la question de la possibilité ou de l'impossibilité de l'ethnographie (Latendresse à paraître ; Makaremi 2016). Cependant, les terrains de la violence 
vont plus loin que de problématiser l'épistémologie ethnographique. En pointant vers le geste parrèsiastique, ils secouent plus profondément encore les fondements de l'anthropologie, voire de notre façon moderne de savoir, renouant de façon irrévocable la question de la vérité à l'éthique de l'action.

\section{Subjectivation, vérité, transformation}

Été 2009. Junaid (nom fictif) est étudiant à l'Institute of Management Science où je travaille à l'époque. Anciennement partie de l'Université de Peshawar, l'institution est située tout juste sur la ligne de partage entre la ville de Peshawar et le district de Khyber (en ce temps-là encore désignée comme zone tribale ou FATA). Le jeune homme cogne un soir à ma porte. Il s'ennuie et cherche quelqu'un à qui faire la conversation. Junaid est originaire du Waziristan-Sud, la zone tribale la plus violente du Pakistan, sous contrôle effectif des Taliban pakistanais. Luniversité est officiellement fermée pour les vacances, mais l'armée mène une opération de contre-insurrection dans quatre des sept « agences » tribales (agencies en anglais, terme administratif désignant les différentes zones tribales du nord-ouest du Pakistan) Junaid et les autres étudiants originaires des FATA clientèle cible de l'établissement - ont une permission spéciale de demeurer sur le campus. Mais même en ville on en est au deuxième attentat-suicide de la semaine (à Peshawar seulement) et forcément la discussion tourne autour de la situation sécuritaire: le couvre-feu, l'armée, qui des « bons » qui des « mauvais » Taliban (une distinction glissante qui me met toujours bien mal à l'aise). Sur le campus les vérités alternatives ne manquent pas: C'est une "guerre contre les Pachtounes ". Ben Laden bien entendu «travaille pour la CIA » contre le Pakistan, et l'armée elle, main dans la main avec le TTP sur lesquels elle tire pourtant depuis des mois. Et selon ces derniers, une campagne de vaccination contre la polio opérée par la Croix rouge est en réalité une tentative de l'Occident de stériliser secrètement les Musulmans. Le personnel médical et les autres volontaires qui y participent sont quotidiennement la cible d'assassinats et d'attentats à la bombe. Ici on ne prend pas les thèses complotistes à la légère. Le plus dérangeant est peut-être de savoir que toutes ces théories, malgré leur bizarrerie et leurs macabres conséquences, ont parfois un inconfortable fond de vérité ${ }^{14}$.

On n'en croit pas la moitié mais, qui sait ce qui se trame vraiment dans ce pays, pas vrai? Junaid tire son portable de sa poche et me dit: « Regarde, voilà ce qui se passe chez moi. ». Il me montre une vidéo. Quelques secondes à peine, mais j'aurais bien cru une éternité. Montés sur des camionnettes, un bataillon du TTP parade sur la route principale à Wanna, exhibant les têtes et les membres coupés de Musulmans Chi'ites de Parachinar, triomphe de leur toute dernière expédition ${ }^{15}$.

Je me rappelle n'avoir pu soutenir la vue de cette vidéo au complet. J'ai fini par craquer et le supplier de l'arrêter. Sur le coup, il était difficile de savoir qui était l'observateur et qui était l'observé. Que signifiait ce sinistre spectacle de jambes, de bras, de têtes, sans corps, comme de vulgaires morceaux de viande sur un étal de boucher (et qui en quelque sorte, était exactement cela)? Dans les mains des Taliban, clairement, ceux-là faisaient office de trophée. Par la vidéo interposée de Junaid, un témoignage. Une dénonciation de l'horreur...

Mais en suis-je vraiment certain? Quoi d'autre aurait-il souhaité communiquer? Cette vidéo, il l'avait prise lui-même. Et pourtant, je restais mystifié par son apparent détachement, le ton laconique de sa voix. Du coup, le jeune homme est devenu comme un jeu de miroir à travers duquel je m'examinais, moi l'anthropologue, sous une multitude d'angles, sans toutefois être sûr de rien saisir. La scène entière - images de chosification extrême de corps humains médiées par une vidéo en trois mégapixels dans les mains d'un jeune homme à l'expression étrangement impassible — devient comme le signifiant d'un énoncé politique polysémique, polyphonique, cacophonique.

Lexpérience de la violence non seulement met au monde un nouveau sujet - comme sujet ethnologique et comme sujet tout court, sa subjectivité altérée et remodelée profondément par l'expérience - mais elle défait et refait également la subjectivité de l'ethnologue lui-même. Elle brise les repères habituels et modifie radicalement son rapport au terrain ethnographique, à la tension éthique-épistémologique qui sous-tend son travail et, ultimement, à lui-même. Tout travail ethnographique comprend, sans nul doute, une large part de remise en question, de son travail, de sa compréhension du monde (il s'agit de l'objectif de la discipline). Mais sur le terrain de la violence, l'incertitude constante, la confusion et la peur remettent plus radicalement encore en cause le rôle, le sens et les dissonances internes du labeur anthropologique.

Ne pouvant échapper aux tiraillements face à l'attraction qu'exerce le risque, la violence, la mort; sur la nécessité (ou non) d'être là pour voir, pour raconter, à défaut de véritablement comprendre, je me mets moi-même en danger - métaphoriquement et littéralement - et je me force à me placer moi-même sous constante observation. «Qu'est-ce qui se passe avec moi? » «En ai-je le droit? » De telles questions ne restent encore qu'en surface, ne touchent encore qu'à des dilemmes moraux, à un problème de légitimité, comme s'il s'agissait de trouver 
réconfort dans leur simple prononciation. Mais une ethnographie de la terreur force à une introspection qui doit aller bien au-delà d'une réflexivité narcissique, comme l'exprime d'ailleurs Mariella Pandolfi (2008) lorsqu'elle parle d' " ethnographie de la désorientation » ou encore, Michael Taussig (1987) par sa notion d' « embrun épistémique ", pour décrire cette expérience ethnographique bien particulière où la relation avec la violence — avec la violence comme objet d'enquête - transforme le rapport au savoir, au connaissable.

Dans L'herméneutique du sujet, Michel Foucault $(2001,28)$ affirme, un peu schématiquement, que le moment déterminant, " où l'histoire de la vérité est entrée dans l'âge moderne $»^{16}$ est celui où l'on a déconnecté « le principe d'un accès à la vérité qui se ferait dans les termes du seul sujet connaissant et, d'autre part, la nécessité spirituelle d'un travail du sujet sur lui-même, se transformant et attendant de la vérité son illumination et sa transfiguration ». Jusqu'à ce grand divorce, ce «tournant » épistémologique décisif - passage de l'alchimie à la chimie moderne - on ne verra jamais ces deux questions que comme inséparables l'une de l'autre. On ne concevra jusque-là l'accès à la vérité qu'à travers un travail sur soi, travail de transformation de soi, comme la promesse d'une vérité transfiguratrice du sujet connaissant.

Or on pourrait se risquer à dire que l'anthropologie contemporaine, et particulièrement l'ethnographie de la violence, par ses préoccupations réflexives sur le processus même du terrain et son effet profondément bouleversant, rouvre de nouveau un espace de questionnement qui rattache l'accès à la vérité à l'exigence d'un travail sur soi du sujet connaissant. Une anthropologie axée sur l'expérience - double, celle du sujet et celle de l'anthropologue à la fois - et qui se maintient en tension perpétuelle entre ses visées autocritiques et ses propres tentations « spiritualisantes ", ou pseudo-thérapeutiques. Cette anthropologie contemporaine tend à se redéfinir comme pratique - au sens où l'importance du processus du terrain en tant que tel semble souvent primer sur les conclusions, et donnent pied à une sorte de philosophie en action plutôt qu'à la recherche de généralisations scientifiques au sens strict.

Les observations de Taussig (1987) sur la façon dont l'imaginaire chamanique exprime la fracture coloniale trouvent écho également chez Eduardo Kohn (2013), dans la «digestion» de ces rapports de pouvoir historiques, telle qu'elle se présente dans l'interprétation des rêves et des présages, voire dans la simple façon de nommer les animaux ou les plantes. Lorsque Elizabeth Povinelli (2016) critique la façon dont le libéralisme tardif déclasse certaines catégories de personnes en fonction d'un principe de séparation (ou non) entre le vivant et le non-vivant, elle ne remet pas en question les fondements de la biologie. Il ne s'agit pas non plus d'établir une sorte de cartographie - réplicable, falsifiable — de ces imaginaires culturels, comme on le ferait de systèmes de parentés ou de règles religieuses. Plutôt de prendre à bras le corps la désorientation dans laquelle nous plonge l'altérité, et cette expérience de la violence (physique, culturelle ou structurelle), afin d'atteindre à une perspective renouvelée.

Les effets de cette épreuve sur le sujet-anthropologue deviennent de façon récurrente l'objet même de l'ethnographie. Une ethnographie de la violence, « ethnographie de la désorientation ", qui remet radicalement en cause la position du chercheur, sa relation au terrain, son accès à la vérité. Elle représente en ce sens la pratique d'une sorte de savoir constituant qui, à la fois, fonde et transfigure le sujet-ethnographe. Un savoir également poursuivi dans l'optique non seulement d'interpréter ce monde qui nous transforme mais aussi (comme disait Marx) de le changer en retour.

Car cette pratique anthropologique tend à se fondre, sous une forme ou une autre dans l'engagement social et politique. Lanthropologie de Michel Agier ou encore de Chowra Makaremi, par exemple, prennent leur sens avant tout dans le contexte d'une lutte politique pour les droits des réfugiés, où les parcours académiques et militants se nourrissent l'un l'autre, comme en parle Makaremi dans ce texte:

\footnotetext{
En zone d'attente, une "participation observante " doit faire face aux conditions propres à l'assistance juridique: sa dimension militante et collective, sa nature légale et sa capacité à intervenir dans le parcours des personnes contrôlées, en leur faveur. Mon implication comme bénévole a précédé, et bien sûr décidé, le projet d'une recherche sur la zone d'attente. Le travail bénévole tendait, d'une part, à faire sortir les étrangers enfermés dans la frontière, et d'autre part, comme effet secondaire, à informer et rendre l'expérience du maintien compréhensible, en créant autant que possible les conditions de réappropriation de la situation vécue. (Makaremi 2008, 167-168)
}

Si tous les anthropologues ne sont directement impliqués (en milieu associatifs, militants, etc.), cette double démarche d'une anthropologie de la violence poursuit néanmoins toujours un même objectif de dépassement: celui de briser l'impuissance de la connaissance pure, et permettre de penser l'action politique au-delà des contraintes institutionnelles et des cadres d'action limités qui régissent le travail des associations, des ONG ou des regroupements politiques.

Ce n'est pas dire qu'elle y parvient. Il n'y a certainement pas de formule, ni de règles d'engagement. Mais 
pour romantique que puisse peut-être paraître cette démarche anthropologie, c'est ici qu'elle trace en réalité le fil rouge qui raccroche la vérité à l'éthique et à un impératif de justice — donc à sa dimension politique. C'est là, justement, tout l'enjeu d'une démarche qui puise son sens dans la parrhésie, qui est « courage de la vérité ». Une vérité qui est avant toute chose justesse (ou justice) de l'acte.

Comme le fait remarquer Franck Chaumon (2009, 153), «le véritable sujet du 'souci de soi' [au centre de L'herméneutique du sujet] n'est pas le sujet de la connaissance, c'est 'l'action droite' ». C'est bien là, en fait, tout le sens de ce "travail sur soi ", de cette exigence transformatrice à laquelle l'accès à la vérité était rattachée. À partir du grand partage entre l'éthique et le savoir, écrit Chaumon, " [l]e sujet de la connaissance vraie de l'Occident moderne a effacé le sujet de l'action droite de l'Antiquité. C'est pourquoi, il faut opérer un retour au logos conçu non pas comme perfection de la connaissance mais comme actualisant la rectitude de l'action. » (153.).

Parce qu'ils dérangent à ce point nos sens, désorientent et nous placent face à nous-mêmes, parfois presqu'à la manière d'une expérience "mystique ", les terrains de la violence, terrains de l'urgence et de la terreur, réorientent radicalement le rapport à soi et à l'autre. Mais de façon plus cruciale encore, ce décentrement de la pratique anthropologique contemporaine, mise en tension entre la théorie et les textures fines de l'expérience vécue, entre épistémologie et éthique, entre transformation « spirituelle » et corporéité, présente le moment crucial d'une reconnexion entre le savoir et l'action. S'il ouvre sur de nouveaux espaces de connaissance, de nouvelles manières de connaitre, ce rapport renouvelé nous aidera peut-être à redéfinir - pour reprendre les mots d'Agamben (2003) « ce que signifie agir politiquement ».

\section{Simon Latendresse, Université de Montréal, Montréal, QC, Canada. Courriel : latendresse.simon. mtl@gmail.com.}

\section{Notes}

1 En 2004, la Banque mondiale a approuvé un prêt de 123 millions USD pour la remodélisation du barrage de Taunsa, construit dans le district de Dera Ghazi Khan au Pendjab, en 1958. Les travaux ont été entrepris en 2005 sans consultation des communautés riveraines et ont donné lieu à l'expulsion sauvage de villages de pêcheurs et à la destruction de terres agricoles dans les localités voisines, menant à un vif mouvement de résistance civile à l'échelle locale.

2 Caste de pêcheurs anciennement semi-nomades mais généralement sédentarisés autour de Taunsa depuis la construction du barrage, en 1958. Considérés comme sindhis par les autorités locales, ils se revendiquent autochtones de la région de Taunsa. De plus, ces pêcheurs, jadis indépendants, ont été soumis à des pratiques de monopolisation de la pêche par des « contracteurs » locaux qui les extorquent et les réduisent au servage.

3 Le film entier sera de toute façon tabletté par l'organisme qui l'avait commandé et coproduit. À court de budget et de temps, ce dernier ne sera jamais présenté au public.

4 Le Mouvement «Sauvez l'Indus » (Sindhu Bachao Tarla, en Séraïki) lutte, entre autres, à travers des manifestations, des actions de désobéissance civile et des grèves de la faim contre les abus des autorités locales et de la Banque mondiale dans la remodélisation du barrage de Taunsa, contre les pratiques prédatrices de nouvelles mafias des pêcheries, ainsi que pour la préservation écologique du bassin de l'Indus.

5 Tehrik-i-Taliban-i-Pakistan (Mouvement Taliban du Pakistan).

6 Je devrais ici spécifier que dans ce texte, je m'interroge exclusivement au sujet l'anthropologie socio-culturelle — et que je désigne aussi parfois, ailleurs dans ce texte par le terme " ethnologie » - et ses dérivés, comme l'anthropologie politique. Sur les branches de l'anthropologie qui se basent principalement sur une méthodologie d'observation participante (ethnologie). Je me préoccupe exclusivement d'une certaine tendance à l'intérieur de celle-ci, mais qui produit néanmoins, depuis plusieurs années, parmi les textes les plus influents et populaires de la discipline. Même si j'emploie fréquemment le terme " anthropologie " tout court, afin d'alléger le texte, il n'est pas ici question d'autres branches de la discipline, telles que l'ethnolinguistique, l'anthropologie physique ou l'archéologie.

7 La courte liste de sources citées ci-haut à propos de l'anthropologie sur la violence et le suicide chez les $\mathrm{Au}$ tochtone n'est d'aucune façon exhaustive et ne sert qu'à la démonstration. Je remercie Aurélie Maire de m'avoir faire remarquer qu'il existe, antérieurement à 2006, des études ethnologiques sur la question.

8 Je prends l'expression « le corps exposé » du titre d'un essai de Didier Fassin « Le corps exposé. Essai d'économie morale de l'illégitimité » (Fassin 2004).

9 Par « image » j'entends ici également la dimension descriptive du témoignage oral ou même de l'écrit.

10 Je laisse sciemment glisser l'une dans l'autre la biopolitique foucaldienne et sa très populaire relecture par Agamben. La première, qui analyse le biopouvoir comme modalité, comme un ensemble immanent de dispositifs de gouvernement; la seconde, comme un rapport souverain à travers l'exercice d'un pouvoir sur la vie biologique (Agamben 1993). C'est que c'est probablement dans le moment de sa contestation que se fait le plus visible le fil rouge qui relie ces deux biopolitiques.

11 Durant la commémoration de l'Achoura, les fidèles « revivent » le martyre de l'Imam Hussain et son deuil, à travers le jeûne et des rituels publics de pleurs et d'autoflagellation. Ces rituels ont souvent tenu lieu de manifestation, projetant leur signification religieuse dans le moment politique du présent — particulièrement lors de la révolution iranienne, durant la guerre Iran-Iraq. Suite à la chute de Saddam Hussein en Iraq, en 2003, Karbala, ville sainte du Chiisme, fut le théâtre, après plusieurs décennies d'interdiction, d'impressionnantes démonstrations de ces rituels collectifs. Au Pakistan, ils ont parfois été interdits et les communautés chi'ites (environ $20 \%$ de la population 
du pays) sont souvent menacées par les groupes sectaires sunnites.

12 Extrait d'un compte rendu anonyme sur Ménestrier, Devise des princes, in Acta eruditorum, 1683.

13 Par «sensibilité biopolitique », j’entends cette intériorisation de la prise en charge, cette régularisation toute moderne de la vie humaine qui, selon Foucault fait répondre à ce pouvoir par ce principe même de " 'droit' à la vie, au corps, à la santé, au bonheur, à la satisfaction des besoins, le 'droit', par-delà toutes les oppressions ou 'aliénations', à retrouver ce qu'on est et tout ce qu'on peut être. " (Foucault 1976, 91).

14 Je ne partage aucunement quelque croyance anti-vaccin que ce soit, encore moins dans cette version aussi extrême. Notons, toutefois, que la stérilisation forcée et massive de femmes pauvres est une pratique ayant lieu encore récemment, dans l'Inde voisine sous Indira Gandhi. Voir le documentaire Something Like a War de Deepa Dhanraj, 1991.

15 Parachinar est une ville du district de Kurrum (anciennement agence tribale fédérale) peuplée en majorité de Pachtounes de confession chi'ites, une très petite minorité religieuse parmi les Pachtounes.

16 Moment que Foucault (2001) ne situe que très vaguement autour du XVII ${ }^{\mathrm{e}}$ siècle.

\section{Références}

Agamben, Giorgio, 1993. Homo Sacer. Le pouvoir souverain et la vie nue. Paris, Seuil.

—, 2003. État d'exception. Paris, Seuil.

Agier, Michel, 2008. Gérer les indésirables. Des camps de réfugiés au gouvernement humanitaire. Paris, Flammarion.

—_, 2012. Réfugiés, sinistrés, sans-papiers. Politiques de l'exception. Paris, Éditions Tétraèdre/Le Sujet dans la Cité.

Agier, Michel (dir.), 1997. Anthropologues en danger : LEngagement sur le terrain. Paris, Éditions Jean-Michel Place.

Benjamin, Walter, 1985 [1928]. Origines du drame baroque allemand. Paris, Flammarion.

__ 2000 [1939]. « L'œuvre d'art à l'époque de sa reproductibilité technique ». Traduit par Maurice de Gandillac. In Walter Benjamin, Euvres III, p. 269-316. Paris, Gallimard.

Bujold, Louise, 2006. La mort habitée : Le suicide chez les jeunes Inuit du Nunavik. Thèse de doctorat, Université Laval.

Chaumon, Franck, 2009. " Michel Foucault, le courage de la vérité ». Essaim 2 (23) : 151-154. https://doi.org/10.3917/ ess.023.0151.

Duffield, Mark, 2000. Global Governance and the New Wars: The Merging of Development and Security. Londres, Zed Books.

Fassin, Didier, 2004. « Le corps exposé. Une économie morale de l'illégitimité ». In Didier Fassin et Dominique Memmi (dir.), Le gouvernement des corps, p. 237-266. Paris, Éditions de l'EHESS.

Foucault, Michel, 1976. Histoire de la sexualité I : La volonté de savoir. Paris, Gallimard

— , 2001. Lherméneutique du sujet. Cours au Collège de France 1981-1982. Paris, Gallimard et Seuil.
—_, 2004. Naissance de la biopolitique. Cours au Collège de France 1978-1979. Paris, Gallimard et Seuil.

—, 2008. Le gouvernement de soi et des autres. Cours au collège de France. 1982-1983. Paris, Gallimard et Seuil.

Eakin, Emily, 2001. « Anthropology's Alternative Radical ». New York Times, 21 avril 2001. https:/www.nytimes. com/2001/04/21/arts/anthropology-s-alternative-radical. html

Giafferi, Natacha, 2004. «Violence de la relation ethnographique. L'exemple haïtien ». Terrain, 43: 123-140. https://doi.org/10.4000/terrain.1864

Grelet, Stanley, et Mathieu Potte-Bonneville, 2000. «Une biopolitique mineure : Entretien avec Giorgio Agamben ». Vacarme, 10. https://vacarme.org/article255.html

Kohn, Eduardo, 2013. How Forests Think: Toward an Anthropology beyond the Human. Berkeley, University of California Press.

Lasvergnas, Isabelle, 2007. « À qui appartient la violence? ». Topique, 99 (2) : 115-127. https://doi.org/10.3917/ top.099.0115.

Latendresse, Simon, À paraître. Sans-papiers à Peshawar : Les ombres de la frontière afghane. Paris, LHarmattan.

Makaremi, Chowra, 2008. "Participer en observant : Étudier et assister les étrangers aux frontières ». In Didier Fassin et Alban Bensa (dir.), Politiques de l'enquête, p. 165-183. Paris, La Découverte.

—_, 2016. " "États d'urgence ethnographiques" : Approches empiriques de la violence politique ". Cultures \& Conflits, 103-104 (3) : 15-34. https://doi.org/10.4000/conflits.19338

Malkki, Liisa, 1995. Purity and Exile: Violence, Memory, and National Cosmology among Hutu Refugees in Tanzania. Chicago, University of Chicago Press.

Mamdani, Mahmood. 2001. When Victims Become Killers: Colonialism, Nativism and Genocide in Rwanda. Princeton, Princeton University Press.

Mbembe, Achille, 2003. " Necropolitics ». Traduit par Libby Meintjes. Public Culture, 15 (1) : 11-40. https://muse.jhu. edu/article/39984. https://doi.org/10.1215/08992363-15-1-11 _ 2018. Politiques de l'inimitié. Paris, La Découverte.

Pandolfi, Mariella, 2008. "Théâtre de guerres : Passions politiques et violences. Anthropologie et Sociétés, 32 (3) : 99-119. https://doi.org/10.7202/029718ar

Povinelli, Elizabeth, 2016. Geontologies: A Requiem to Late Liberalism. Durham, NC, Duke University Press.

Schmitt, Betina E., et Ingo W. Schroeder (dir.), 2001. Anthropology of Violence and Conflict. Londres et New York, Routledge.

Solomon-Godeau, Abigail, 2010. " Photographier la catastrophe ». Terrain, 54: 56-65. https://doi.org/10.4000/ terrain.13962

Taussig, Michael, 1987. Shamanism, Colonialism and the Wild Man: A Study in Terror and Healing. Chicago, University of Chicago Press.

— 1992. The Nervous System. New York, Routledge.

- 2003. Law in a Lawless Land: Diary of a Limpieza in Colombia. Chicago, University of Chicago Press.

Viventi, Livía. 2011. Couper le fil de la vie: Suicide et rituels de mort chez les Atikamekw de Manawan. Thèse de doctorat, Université de Montréal. 